\title{
CRÓNICA PARLAMENTARIA DEL SENADO
}

MARÍA VICTORIA GARCÍA ATANCE GARCÍA DE MORA

Profesora Titular de Derecho Constitucional UNED 


\section{SUMARIO}

B) Competencia de control. B.I. Mociones de Comisión. B.Il. Mociones ante el Pleno. B.III. Interpelaciones. C) ReLACIONES CON ÓRganos E INSTITUciones públicas: con el Tribunal Constitucional. C.I. Cuestiones de inconstitucionalidad. 
Revista de Derecho Político, núm. 50, 2001, págs. 291-309

\title{
CRÓNICA PARLAMENTARIA DEL SENADO
}

\author{
POR \\ MARÍA VICTORIA GARCIAA-ATANCE GARCÍA DE MORA \\ Profesora Titular de Derecho Constitucional. UNED
}

A continuación se refleja la actividad del Senado durante una parte del segundo periodo de sesiones de la VII Legislatura (desde mediados de octubre del año 2000 hasta diciembre de 2000), cerrando con ello el segundo periodo de sesiones, que no había sido reflejado en su totalidad en la crónica correspondiente a la Cámara del Senado del número anterior de la Revista. Por este motivo, sólo se reseñarán a continuación los documentos correspondientes a la competencia de control de la Cámara relativa a las Mociones en Comisión y ante el Pleno; asi como las interpelaciones presentadas. De igual modo, se reflejan las Cuestiones de inconstitucionalidad, propias de las relaciones de la Cámara con otras instituciones públicas, dando con ello por cerrado el segundo periodo de sesiones referenciado.

\section{B) COMPETENCIA DE CONTROL}

\section{B.I. Mociones en Comisión}

De entre las Mociones en Comisión presentadas en la Cámara del Senado, procedemos al desglose de las siguientes y sin que ello implique una relación exhaustiva.

Procedimiento: Ordinario

Fecha de presentación: 8-06-00 


\section{Autor: GRUPO PARLAMENTARIO SOCIALISTA}

Objeto: Moción por la que se insta al Gobierno a que autorice al Instituto para la Reestructuración de la Minería del Carbón y Desarrollo Alternativo de las Comarcas Mineras a suscribir el correspondiente convenio específico para la financiación del proyecto de construcción de una residencia universitaria presentado por el Ayuntamiento de Bélmez (Córdoba).

Situación actual: Pendiente de reunión de Comisión.

Comisión competente: Comisión de Economía, Comercio y Turismo.

Datos de tramitación:

Fecha de entrada en la Comisión: 26-julio-2000

Procedimiento: Ordinario

Fecha de presentación: 14-06-2000

Autor: GRUPO PARLAMENTARIO SOCIALISTA.

Objeto: Moción por la que se insta al Ministerio de Educación, Cultura y Deporte a que realice las gestiones oportunas para apoyar la declaración, por parte de la Organización de las Naciones Unidas para la Educación, la Ciencia y la Cultura (UNESCO), de la ruta de la arquitectura negra en la provincia de Guadalajara como Patrimonio de la Humanidad.

Situación actual: Concluido (Aprobado con modificaciones) en fecha 19-octubre-2000.

Comisión competente: Comisión de Educación, Cultura y Deporte.

Datos de tramitación:

Fecha de entrada en la Comisión: 29-junio-2000

Tramitación: 19-oct.-2000 Debate en Comisión.

Procedimiento: Ordinario

Fecha de presentación: 14-06-00

Autor: GRUPO PARLAMENTARIO SOCIALISTA

Objeto: Moción por la que se insta al Gobierno a que la obra de acondicionamiento del tramo de la carretera nacional N-432, comprendido entre Espiel y la Cuesta de la Matanza (Córdoba), figure entre las actuaciones a ejecutar por el Ministerio de Fomento 
y financiadas con cargo a los presupuestos Generales del Estado para 2001.

Situación actual: Concluido (rechazado) en fecha 18-octubre-2000.

Comisión competente: Comisión de Infraestructuras.

Datos de tramitación:

Fecha de entrada en la Comisión: 29-junio-2000

Tramitación: 18-octubre-2000. Debate en Comisión.

Procedimiento: Ordinario

Fecha de presentación: 28-6-2000

Autor: GRUPO PARLAMENTARIO SOCIALISTA

Objeto: Moción por la que se insta al Gobierno a impulsar, en el seno de la Comisión Mixta de Transferencias, las negociaciones entre la Diputación Foral de Navarra y el Estado para la cesión gratuita de la nuda propiedad de todo el territorio de las Bárdenas Reales a la Comunidad Foral de Navarra, con anterioridad a la realización de cualquier actuación relacionada con el polígono de tiro.

Situación actual: Pendiente de reunión de Comisión.

Comisión competente: Comisión General de las Comunidades Autónomas.

Datos de tramitación:

Fecha de entrada en la Comisión: 25 julio 2000

Procedimiento: Ordinario

Fecha de presentación: 18-07-00

Autor: GRUPO PARLAMENTARIO MIXTO.

Objeto: Moción por la que se insta al Gobierno para que la Ley de acompañamiento de los Presupuestos Generales del Estado para el año 2001 contemple, entre los criterios para el reparto de la participación de los ayuntamientos en los ingresos del Estado, los factores de dispersión de la población, el número de núcleos dentro del término municipal y la extensión del mismo; así como para que dichos criterios sean también tenidos en cuenta en la redacción de la nueva Ley de Haciendas Locales.

Situación actual: Pendiente de reunión de Comisión. 
Comisión competente: Comisión de Economía, Comercio y Turismo.

Datos de tramitación:

Fecha de entrada en la Comisión: 14- sep.-2000

Procedimiento: Ordinario

Fecha de presentación: 18-07-00

Autor: GRUPO PARLAMENTARIO SOCIALISTA

Objeto: Moción por la que se insta al Ministerio de Sanidad y Consumo a que habilite los recursos humanos y materiales necesarios para la creación de un Centro de Especialidades, Diagnóstico y Tratamiento (CEDT) con sede en el centro de salud de Azuqueca de Henares (Guadalajara), a fin de prestar atención a la zona básica de salud.

Situación actual: Pendiente de entrada en Comisión

Procedimiento: Ordinario

Fecha de presentación: 5-09-00

Autor: GRUPO PARLAMENTARIO MIXTO

Objeto: Moción por la que se insta al Gobierno a que haga una memoria de las fincas agrícolas ubicadas en territorio andaluz y actualmente propiedad del Patrimonio del Estado, que fueron expropiadas por el Gobierno al grupo Rumasa, S.A., así como a que proceda a transferir dichas fincas al patrimonio público de la Junta de Andalucía.

Situación actual: Pendiente de entrada en Comisión.

Procedimiento: Ordinario

Fecha de presentación: 18-09-00

Autor: GRUPO PARLAMENTARIO ENTESA CATALANA DE PROGRÉS

Objeto: Moción por la que se insta al Gobierno a proceder a la modifi-. cación de la Orden Ministerial de 15 de septiembre de 2000, relativa a la regulación de las matrículas de automóviles para que incluyan un distintivo que reconozca y refleje el carácter autonómico del Estado.

Situación actual: Pendiente de reunión de Comisión. 
Comisión competente: Comisión General de las Comunidades Autónomas.

Datos de tramitación:

Fecha de entrada en la Comisión: 29 sep. 2000

Procedimiento: Ordinario

Fecha de presentación: 18-09-00

Autor: GRUPO PARLAMENTARIO POPULAR EN EL SENADO

Objeto: Moción por la que se insta al Gobierno a perfeccionar el seguro agrario aumentando la cobertura del riesgo frente a las catástrofes naturales y a las inclemencias del clima, incluyendo nuevas coberturas.

Situación actual: Pendiente de reunión de Comisión

Comisión competente: Comisión de Agricultura, Ganadería, Pesca y Alimentación.

Datos de tramitación:

Fecha de entrada en la Comisión: 29 sept. 2000

\section{B.II. Mociones ante el Pleno}

Procedimiento: Ordinario

Fecha de presentación: 22-05-00

Autor: GRUPO PARLAMENTARIO POPULAR EN EL SENADO.

Objeto: Moción por la que se insta al Gobierno a que en el plazo de seis meses elabore un proyecto de ley de reforma del Código Civil que regule, en los procesos que resuelvan crisis de parejas, la posibilidad de que los hijos continúen su comunicación y relación de afectividad con sus abuelos, recabando al efecto el dictamen de especialistas.

Situación actual: Concluido (Aprobado con modificaciones) en fecha 30 mayo 2000.

Datos de tramitación:

Tramitación: 22 mayo 2000 Calificación por la Presidenta 30 mayo 2000 Sesión del Pleno

N. de enmiendas: 1 
N.ำ de propuestas de modificación: 1

Procedimiento: Ordinario

Fecha de presentación: 11-04-00

Autor: GRUPO PARLAMENTARIO CATALÁN EN EL SENADO DE CONVERGENCIA I UNIÓ

Objeto: Moción por la que se insta a la Cámara a impulsar, con la mayor celeridad posible, una reforma del Reglamento del Senado al objeto de posibilitar la utilización, en todas las sesiones del mismo, de cualquiera de las lenguas que tengan el carácter de oficial en alguna Comunidad Autónoma.

Situación actual: Concluido (Retirado) en fecha 27 abril 2000

Procedimiento: Ordinario

Fecha de presentación: 27-04-00

Autor: GRUPO PARLAMENTARIO CATALÁN EN EL SENADO DE CONVERGÈNCIA I UNIÓ

Objeto: Moción para que el Pleno del Senado impulse una reforma del Reglamento para hacer posible la utilización, en todas las sesiones del mismo, de cualquiera de las lenguas que tengan el carácter de oficial en alguna Comunidad Autónoma.

Situación actual: Concluido (Aprobado con modificaciones) en fecha 16 mayo 2000

Datos de tramitación:

Tramitación: 09 mayo 2000 Calificación por la Presidenta

16 mayo 2000 Sesión del Pleno

NN. de enmiendas: 4

N.․ de propuestas de modificación: 1

Procedimiento: Ordinario

Fecha de presentación: 4-05-00

Autor: GRUPO PARLAMENTARIO CATALÁN EN EL SENADO DE CONVERGENCIA I UNIÓ. 
Objeto: Moción por la que se insta al Gobierno a ampliar las medidas compensatorias para los sectores agrario y pesquero como consecuencia del encarecimiento del precio del gasóleo destinado a estas actividades.

Situación actual: Concluido (Decaído) en fecha 09 mayo 2000

Datos de tramitación:

Tramitación: 09 mayo2000 Calificación por la Presidenta

Procedimiento: Ordinario

Fecha de presentación: 5-05-00

Autor: GRUPO PARLAMENTARIO CATALÁN EN EL SENADO DE CONVERGENCIA I UNIÓ

Objeto: Moción por la que se insta al Gobierno a que defienda ante las instituciones de la Unión Europea la reforma de la Organización Común de Mercado (OCM) de frutas y hortalizas, y a que mantenga una ayuda a la renta de los productores hasta que dicha reforma no se haga efectiva.

Situación actual: Concluido (Decaído) en fecha 9 mayo 2000

Datos de tramitación:

Tramitación: 9 mayo 2000 Calificación por la Presidenta

Procedimiento: Ordinario

Fecha de presentación: 8-05-00

Autor: GRUPO PARLAMENTARIO SOCIALISTA.

Objeto: Moción por la que se insta al Gobierno para que los titulares de cada Ministerio comparezcan ante la Comisión correspondiente de la Cámara y expongan los programas de actuación de sus respectivos Departamentos, especialmente en lo que concierne al desarrollo del Estado de las Autonomías.

Situación actual: Concluido (Aprobado con modificaciones) en fecha 16 mayo 2000

Datos de tramitación:

Tramitación: 9 mayo 2000 Calificación por la Presidenta 16 de Mayo 2000 Sesión del Pleno

N. de enmiendas: 1 
Procedimiento: Ordinario

Fecha de presentación: 8-05-00

Autor: GRUPO PARLAMENTARIO POPULAR EN EL SENADO.

Objeto: Moción por la que se propone la creación de una ponencia para reformar el debate sobre el Estado de las Autonomías, asi como aquellos aspectos del Reglamento del Senado que mejoren su eficacia en el ejercicio de las funciones que, como Cámara de representación territorial, le otorga la Constitución.

Situación actual: Concluido (Aprobado con modificaciones) en fecha 16 mayo 2000.

Datos de tramitación:

Tramitación: 9 mayo 2000 Calificación por la Presidenta

16 mayo 2000 Sesión del Pleno

N.ㅇ de enmiendas: 5

N. de propuestas de modificación: 1

Procedimiento: Ordinario

Fecha de presentación: 8-5-00

Autor: GRUPO PARLAMENTARIO POPULAR EN EL SENADO

Objeto: Moción por la que se insta a la utilización por parte del Senado de las nuevas tecnologías de la información como herramienta para la participación de todos los españoles en las actividades que se desarrollen en la Cámara.

Situación actual: Concluido (Aprobado con modificaciones) en fecha 16 mayo 2000.

Datos de tramitación:

Tramitación: 9 mayo 2000 Calificación por la Presidenta

16 mayo 2000 Sesión del Pleno

N.․ de enmiendas: 2

N.ำ de propuestas de modificación: 1

Procedimiento: Ordinario

Fecha de presentación: 19-5-00 
Autor: GRUPO PARLAMENTARIO CATALÁN EN EL SENADO DE CONVERGĖNCIA I UNIÓ

Objeto: Moción por la que se insta al Gobierno a que defienda ante las instituciones de la Unión Europea la reforma de la Organización Común de Mercado (OCM) de frutas y hortalizas, y a que mantenga una ayuda a la renta de los productores hasta que dicha reforma no se haga efectiva.

Situación actual: Concluido (Decaído) en fecha 22 mayo 2000

Datos de tramitación:

Tramitación: 22 mayo 2000 Calificación por la Presidenta

\section{Procedimiento: Ordinario}

Fecha de presentación: 22-05-00

Autor: GRUPO PARLAMENTARIO SOCIALISTA

Objeto: Moción por la que se insta al Gobierno a presentar ante la Comisión General de las Comunidades Autónomas un informe sobre la cuantificación de los fondos previstos para garantizar la capacidad de cobertura de la demanda de servicios públicos para el quinquenio 1997-2001, así como a adoptar medidas para que las Comunidades Autónomas que sean beneficiarias de la garantía de capacidad de cobertura de la demanda de servicios públicos, dispongan este mismo año de un anticipo de tesorería a cuenta de la aplicación de la garantía en el año 2000.

Situación actual: Concluido (Rechazado) en fecha 30-mayo-2000

Datos de tramitación:

Tramitación: 22 mayo 2000 Calificación por la Presidenta 30 mayo 2000 Sesión del Pleno

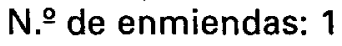

Procedimiento: Ordinario

Fecha de presentación: 22-05-00

Autor: GRUPO PARLAMENTARIO POPULAR EN EL SENADO.

Objeto: Moción por la que se insta al Gobierno a poner en práctica todas aquellas medidas que hagan posible romper las barreras 
que existen para lograr la integración definitiva y el acceso en condiciones de igualdad de la mujer en la vida laboral.

Situación actual: Concluido (Aprobado sin modificaciones) en fecha 30 mayo 2000

Datos de tramitación:

Tramitación: 22 mayo 2000 Calificación por la Presidenta

30 mayo 2000 Sesión del Pleno

N. ${ }^{\circ}$ de enmiendas: 3

Procedimiento: Ordinario

Fecha de presentación: 8-06-00

Autor: GRUPO PARLAMENTARIO ENTESA CATALANA DE PROGRÉS

Objeto: Moción por la que se insta al Gobierno a modificar el Real Decreto 1186/1998, al objeto de adecuar su contenido a las necesidades actuales de acceso a la vivienda en regímenes protegidos.

Situación actual: Concluido (Decaído) en fecha 20 junio 2000

Datos de tramitación:

Tramitación: 20 junio 2000 Calificación por la Presidenta

Procedimiento: Ordinario

Fecha de presentación: 14-07-00

Autor: GRUPO PARLAMENTARIO MIXTO

Objeto: Moción por la que se insta al Gobierno a suprimir la determinación totalmente libre de los horarios de los establecimientos comerciales y a atribuir a las Comunidades Autónomas el ejercicio en exclusiva de dicha competencia.

Situación actual: Concluido (Rechazado) en fecha 12 septiembre 2000 Datos de tramitación:

Tramitación: 5 sep. 2000 Calificación por la Presidenta 12 sep. 2000 Sesión del Pleno

N. de propuestas de modificación: 1

Procedimiento: Ordinario

Fecha de presentación: 19-07-00 
Autor: GRUPO PARLAMENTARIO MIXTO

Objeto: Moción por la que se insta al Gobierno a la creación de una comisión de estudio para la puesta en marcha de una tasa sobre las transacciones especulativas en los mercados de divisas.

Situación actual: Concluido (Decaído) en fecha 5 sep. 2000

Datos de tramitación:

Tramitación: 5 sep. 2000 Calificación por la Presidenta

Procedimiento: Ordinario

Fecha de presentación: 1-09-00

Autor: GRUPO PARLAMENTARIO ENTESA CATALANA DE PROGRÉS

Objeto: Moción por la que se acuerda la creación de una ponencia de estudio y seguimiento del sistema de financiación autonómica, en el seno de la Comisión General de las Comunidades Autónomas, así como por la que se acuerda estudiar, a través de dicha Comisión, la conveniencia de crear una oficina técnica de datos económico-territoriales.

Situación actual: Concluido (Decaído) en fecha 5 sep. 2000

Datos de tramitación:

Tramitación: 5 sep. 2000 Calificación por la Presidenta

Procedimiento: Ordinario

Fecha de presentación: 4-09-00

Autor: GRUPO PARLAMENTARIO POPULAR EN EL SENADO

Objeto: Moción por la que se insta al Gobierno a elaborar un código de consumo que aporte claridad, coherencia y facilidad en el acceso a la dispersa y abundante normativa que sobre consumo existe en la actualidad.

Situación actual: Concluido (Aprobado con modificaciones) en fecha 12 sep. 2000.

Datos de tramitación:

Tramitación: 5 sep. 2000 Calificación por la Presidenta

12 sept. 2000 Sesión del Pleno

N.ㅇ de enmiendas: 4 


\section{Procedimiento: Ordinario}

Fecha de presentación: 11-09-00

Autor: GRUPO PARLAMENTARIO ENTESA CATALANA DE PROGRÉS

Objeto: Moción por la que se acuerda la creación de una ponencia de estudio y seguimiento del sistema de financiación autonómica, en el seno de la Comisión General de las Comunidades Autónomas, así como por la que se acuerda estudiar, a través de dicha Comisión, la conveniencia de crear una oficina técnica de datos económico-territoriales

Situación actual: Concluido (Decaído) en fecha 19 sep. 2000

Datos de tramitación:

Tramitación: 19 sep. 2000 Calificación por la Presidenta

Procedimiento: Ordinario

Fecha de presentación: 12-09-00

Autor: GRUPO PARLAMENTARIO MIXTO

Objeto: Moción por la que se insta al Gobierno a la creación de una comisión de estudio para la puesta en marcha de una tasa sobre las transacciones especulativas en los mercados de divisas.

Situación actual: Concluido (Decaido) en fecha 19 sep. 2000

Datos de tramitación:

Tramitación: 19 sep. 2000 Calificación por la Presidenta

\section{B.III. Interpelaciones}

Procedimiento: Ordinario

Fecha de presentación: 3-05-00

Autor: ARMET I COMA, LLUÍS (GPECP)

Objeto: Interpelación sobre la política que se propone desarrollar el Gobierno al servicio del pluralismo territorial.

Situación actual: Concluido (Formulado Pleno) en fecha 17 mayo 2000 Datos de tramitación:

Tramitación: 09 mayo 2000 Calificación por la Presidenta

17 mayo 2000 Sesión del Pleno 
Procedimiento: Ordinario

Fecha de presentación: 8-05-00

Autor: LABORDA MARTIN, JUAN JOSÉ (GPS)

Objeto: Interpelación sobre la política autonómica del Gobierno y la falta de compromisos para desarrollarla parlamentariamente en el Senado.

Situación actual: Concluido (Formulado Pleno) en fecha 17 mayo 2000 Datos de tramitación:

Tramitación: 09 mayo 2000 Calificación por la Presidenta 17 mayo 2000 Sesión del Pleno

Procedimiento: Ordinario

Fecha de presentación: 1-06-00

Autor: RÍOS PÉREZ, VICTORIANO (GPCC)

Objeto: Interpelación sobre el grado de cumplimiento del artículo 38.1 del vigente Estatuto de Autonomía de Canarias y el de otros que contengan normativas similares, referentes a la información previa a las Comunidades Autónomas en la elaboración de los Tratados y Convenios Internacionales y en las negociaciones de adhesión a los mismos.

Situación actual: Concluido (Formulado Pleno) en fecha 28 junio 2000 Datos de tramitación:

Tramitación: 13 junio 2000 Aplazamiento por el Gobierno 20 junio 2000 Calificación por la Presidenta 28 junio 2000 Sesión del Pleno

Procedimiento: Ordinario

Fecha de presentación: 2-06-00

Autor: VARELA I SERRA, JOSEP (GPCIU)

Objeto: Interpelación sobre la política y el calendario previsto por el Gobierno para la implantación en España de una tarifa plana asequible y universal para los usuarios de internet.

Situación actual: Concluido (Formulado Pleno) en fecha 14 junio 2000 
Datos de tramitación:

Tramitación: 6 junio 2000 Calificación por la Presidenta

14 junio 2000 Sesión del Pleno

Procedimiento: Ordinario

Fecha de presentación: 4-07-00

Autor: VARELA I SERRA, JOSEP (GPCIU)

Objeto: Interpelación sobre la política que piensa llevar a cabo el Gobierno para proteger a los sectores empresariales ligados con

el libro, así como para fomentar el hábito de lectura en el país.

Situación actual: Concluido (Formulado Pleno) en fecha 13 sep. 2000

Datos de tramitación:

Tramitación: 05 sep. 2000 Calificación por la Presidenta 13 sep. 2000 Sesión del Pleno

Procedimiento: Ordinario

Fecha de presentación: 4-09-00

Autor: ARNAU NAVARRO, FRANCISCO (GPS)

Objeto: Interpelación sobre la expedición de altas médicas en los procesos de incapacidad temporal por parte de los médicos de las mutuas de accidentes de trabajo y enfermedades profesionales.

Situación actual: Concluido (Formulado Pleno) en fecha 13 sept. 2000 Datos de tramitación:

Tramitación: 05 sept. 2000 Calificación por la Presidenta 13 sep. 2000 Sesión del Pleno

Procedimiento: Ordinario

Fecha de presentación: 18-09-00

Autor: LOPEZ CARRASCO, FERNANDO (GPS)

Objeto: Interpelación sobre la política prevista por el Gobierno para corregir el descenso de las rentas de los agricultores y ganaderos.

Situación actual: Concluido (Formulado Pleno) en fecha 27 sep. 2000 
Datos de tramitación:

Tramitación: 19 sept. 2000 Calificación por la Presidenta

27 sept. 2000 Sesión del Pleno

Procedimiento: Ordinario

Fecha de presentación: 28-09-00

Autor: MARIMON I SABATÉ, FRANCESC XAVIER (GPCIU)

Objeto: Interpelación sobre las medidas que piensa adoptar el Gobierno para suprimir los problemas que genera la burocracia en la exportación de la industria cárnica.

Situación actual: Concluido (Formulado Pleno) en fecha 11 octubre 2000

Datos de tramitación:

Tramitación: 03 oct. 2000 Calificación por la Presidenta

11 oct. 2000 Sesión del Pleno

Procedimiento: Ordinario

Fecha de presentación: 28-09-00

Autor: CABRERO PALOMARES, JOSÉ (GPMX)

Objeto: Interpelación sobre la política del Gobierno en relación con el establecimiento de mecanismos democráticos de regulación $y$ control de los mercados y del sistema financiero.

Situación actual: Concluido (Formulado Pleno) en fecha 11 octubre 2000

Datos de tramitación:

Tramitación: 03 oct. 2000. Calificación por la Presidenta

11 oct. 2000 Sesión del Pleno

Procedimiento: Ordinario

Fecha de presentación: 28-09-00

Autor: QUINTANA GONZÁLEZ, ANXO MANUEL (GPMX)

Objeto: Interpelación sobre las medidas diseñadas por el Gobierno para dar cumplimiento al Plan de Fomento de las Energías Renovables. 
Situación actual: Concluido (Decaído) en fecha 3 octubre 2000 Datos de tramitación:

Tramitación: 03 oct. 2000 Calificación por la Presidenta

C) RELACIONES CON ÓRGANOS E INSTITUCIONES PÚBLICAS: CON EL TRIBUNAL CONSTITUCIONAL

\section{C.I. Cuestiones de inconstitucionalidad}

Procedimiento: Ordinario

Fecha de presentación: 25/09/00

Autor: TRIBUNAL CONSTITUCIONAL

Objeto: Cuestión de inconstitucionalidad número 4891/1999, planteada por la Sala de lo Social del Tribunal Superior de Justicia de las Islas Baleares, en relación con el artículo 6.5 de la Ley de la Comunidad Autónoma de las llles Balears 5/1996, de 18 de diciembre, de Medidas Tributarias y Administrativas.

Situación actual: Concluido (Tramitado) en fecha 03 Oct 2000.

\section{Procedimiento: Ordinario}

Fecha de presentación: 25/09/00

Autor: TRIBUNAL CONSTITUCIONAL

Objeto: Cuestión de inconstitucionalidad número 2861/2000, planteada por la Sala de lo Contencioso-Administrativo del Tribunal Superior de Justicia de Extremadura, en relación con la Disposición Transitoria Segunda de la Ley de Extremadura 3/1996, de 25 de junio, de Atención Farmacéutica de la Comunidad Autónoma.

Situación actual: Concluido (Tramitado) en fecha 03 Oct 2000.

\section{Procedimiento: Ordinario}

Fecha de presentación: 26/09/00

Autor: TRIBUNAL CONSTITUCIONAL

Objeto: Cuestión de inconstitucionalidad número 1378/2000, planteada por la Sección Primera de la Sala de lo Contencioso-Administrativo del Tribunal Superior de Justicia de Cataluña, por supues- 
ta inconstitucionalidad del artículo 9.7 del Decreto 3313/1966, que aprueba el Texto Refundido de la Ley del Impuesto sobre Actividades y Beneficios Comerciales e Industriales; y del ejercicio de la delegación legislativa contenido en el artículo 279.7 y en la Disposición derogatoria undécima, apartado 1, del Real DecretoLegislativo 781/1986, de 18 de abril, por el que se aprueba el Texto Refundido de las disposiciones legales vigentes en materia de régimen local.

Situación actual: Concluido (Tramitado) en fecha 03 Oct 2000. 